\title{
Clinical course of COVID-19 in patients with pre-existing decompensated cirrhosis: initial report from China
}

\author{
Xiaolong $\mathrm{Qi}^{1}{ }^{10} \cdot$ Jitao Wang ${ }^{1} \cdot$ Xinyu $\mathrm{Li}^{2} \cdot$ Zhengyan Wang $^{3} \cdot$ Yanna Liu ${ }^{1} \cdot$ Hua Yang ${ }^{4} \cdot$ Xiaodan $\mathrm{Li}^{5} \cdot$ Jindong Shi $^{6}$. \\ Huihua Xiang ${ }^{7} \cdot$ Tao Liu $^{5} \cdot$ Norifumi Kawada $^{8} \cdot$ Hitoshi Maruyama ${ }^{9} \cdot$ Zicheng Jiang $^{10}$. Fengmei Wang ${ }^{11}$. \\ Tetsuo Takehara ${ }^{12}$. Don C. Rockey ${ }^{13}$. Shiv Kumar Sarin ${ }^{14} \cdot$ for the COVID-Cirrhosis-CHESS Group
}

Received: 16 April 2020 / Accepted: 4 May 2020 / Published online: 22 May 2020

(c) Asian Pacific Association for the Study of the Liver 2020

\begin{abstract}
Background The clinical characteristics and disease course in COVID-19 patients with pre-existing decompensated cirrhosis has not been described so far.

Methods In this case series, we report three patients with confirmed COVID-19 and pre-existing decompensated cirrhosis from three hospitals in Hubei, the epicenter of the outbreak in China.

Result Patient 1 was a 53-year-old man with hepatitis B virus-related cirrhosis, portal hypertension, and ascites. Though receiving intensive support, he died of irreversible multiple organ dysfunction syndrome 48 days after the onset of the illness. Patient 2 was a 75-year-old woman with a history of schistosomiasis-related cirrhosis, portal hypertension, and ascites. Her family members requested that invasive rescue measures not be undertaken, and she died of acute respiratory distress syndrome 40 days after presenting with COVID-19 infection. Patient 3 was an 87-year-old man with alcohol-related cirrhosis, portal hypertension, and esophageal variceal hemorrhage. He was discharged from the hospital 29 days after illness onset. Conclusion The case series raise the possibility that decompensated cirrhosis may be a risk factor for a poor outcome in patients with COVID-19.
\end{abstract}

Keywords COVID-19 Advanced chronic liver disease $\cdot$ Clinical characteristics

\section{Introduction}

The rapidly expanding coronavirus disease 2019 (COVID19) pandemic has had a major impact on the medical care of many individuals throughout the world [1,2]. Previous studies have reported that patients with severe COVID-19 disease have abnormal aminotransferase levels, which raise the possibility of significant COVID-19-induced liver injury $[3,4]$. This clinical challenge is even prominent in patients with COVID-19 and pre-existing decompensated cirrhosis - in particular, considering their immunocompromised

Xiaolong Qi, Jitao Wang, Xinyu Li and Zhengyan Wang Joint first authors.

Xiaolong Qi, Don C. Rockey and Shiv Kumar Sarin are Joint senior authors.

Xiaolong Qi

qixiaolong@vip.163.com

Extended author information available on the last page of the article status and worse clinical outcomes than compensated cirrhosis $[4,5]$. Acute exacerbation of liver function has been described in decompensated cirrhosis clinical guidelines, although the effects of COVID-19 are not well defined [5, 6]. Since the clinical characteristics and disease course in COVID-19 patients with pre-existing decompensated cirrhosis are incompletely defined, in an effort to better understand this clinical scenario, we herein report three cases of patients with laboratory-confirmed COVID-19 and pre-existing decompensated cirrhosis from three hospitals in Hubei, the epicenter of the outbreak in China.

\section{Case series}

A case of COVID-19 was defined as a confirmed positive after one of the following results: (1) demonstration of the presence of severe acute respiratory syndrome coronavirus 2 (SARS-CoV-2) with high-throughput sequencing from nasopharyngeal swab specimens, (2) a positive real-time 
reverse-transcriptase polymerase-chain-reaction assay from nasopharyngeal swab specimens, or (3) the demonstration of SARS-CoV-2 specific IgM and IgG in serum [1-3]. Decompensated cirrhosis was defined as clinically or histologically diagnosed cirrhosis with a history of at least one decompensating event, such as ascites, hepatic encephalopathy, and/or variceal hemorrhage.

\section{Clinical characteristics and laboratory results at admission}

\section{Patient 1}

A 53-year-old man with hepatitis B virus-related cirrhosis (Child-Pugh class C; Model for End-stage Liver Disease [MELD] score, 15) was diagnosed with decompensated cirrhosis after presenting with ascites previously and has been treated with entecavir. He experienced his first symptoms (fever, cough, sputum, dizziness, and myalgia) on 22 January 2020 after coming back from Wuhan (Hubei, China), and was admitted to the hospital on 25 January 2020 with pneumonia diagnosed by chest $\mathrm{CT}$ imaging. The diagnosis of COVID-19 was confirmed on 28 January 2020. Laboratory results on admission showed leukocytosis, lymphopenia and thrombocytopenia, hyperbilirubinemia, hypoproteinemia and prolonged prothrombin time (Table 1). Alanine aminotransferase (ALT), and alkaline phosphatase (ALP) levels were in the normal range; and aspartate transaminase (AST) and gamma-glutamyl transferase (GGT) levels were slightly elevated (Table 1). Chest CT showed bilateral ground-glass opacities, typical of pulmonary COVID-19 infection.

\section{Patient 2}

A 75-year-old woman with a 5-year history of cirrhosis (Child-Pugh class C; MELD score, 14) due to schistosomiasis and was diagnosed with decompensated cirrhosis approximately 6 months previously after presenting with ascites. She was admitted to the hospital on 12 March 2020 after 30 days of intermittent fever after coming back from Wuhan (Hubei, China); and was confirmed with COVID-19 on 12 March 2020. Laboratory results at admission revealed leukopenia, lymphopenia and thrombocytopenia (Table 1). In addition, ALT and AST levels were both minimally elevated and GGT and ALP levels were elevated (Table 1). Chest CT scan revealed ground-glass opacities in the left lung, typical of pulmonary COVID-19 infection.

\section{Patient 3}

An 87-year-old man with a 7-year history of alcoholrelated cirrhosis (Child-Pugh class, B; MELD score, 23) was diagnosed with decompensated cirrhosis approximately 7 years previously after presenting with variceal hemorrhage. He reported a close contact with sick persons several days before he developed cough, white frothy sputum, diarrhea and fever. He was confirmed to have COVID-19 and admitted to the intensive care unit (ICU) on 11 February 2020. Laboratory results on admission showed leukocytosis, lymphopenia and thrombocytopenia, hyperbilirubinemia, hypoproteinemia and prolonged prothrombin time (Table 1). ALT level was normal, while AST and GGT levels were slightly elevated. Chest CT revealed bilateral ground-glass opacities and large bilateral pleural effusions.

\section{Treatment, complications and outcomes}

Patients 1 and 2 deteriorated clinically on 2 February 2020 (day 12 from the onset of the illness) and 20 March 2020 (day 38 from the onset of the illness), respectively, and were transferred to the ICU (Table 2). All patients were given antiviral therapy (patient 1: lopinavir plus ritonavir orlly [400 mg/100 mg, BID] combined with inhalation of interferon alfa-2b [500 wu, BID]; patient 2: arbidol orally [200 mg, TID]; patient 3: lopinavir plus ritonavir orally [400 mg/100 mg, BID] combined with arbidol orally [200 mg, TID] and with a switch to inhalation of interferon alfa-2b [500 wu, BID] because of an ongoing increase in bilirubin). In addition, all patients received liver protective drugs (glutathione-based therapy), broad spectrum antibiotics, systemic glucocorticoids, intravenous immunoglobulin therapy and intravenous blood products, such as plasma, albumin and cryoprecipitate. During hospitalization, all developed ascites and patients 1 and 2 developed pleural effusions. Therapeutic paracentesis was performed in all patients, as well as therapeutic thoracentesis in patient 1 . Patient 1 and 3 developed melena and were treated with a proton pump inhibitor, somatostatin, and red blood cell transfusion to a target hemoglobin level of $8 \mathrm{mg} / \mathrm{dL}$.

Patient 1 required endotracheal intubation and developed acute kidney injury, requiring continuous renal replacement therapy and extracorporeal membrane oxygenation. $\mathrm{He}$ developed progressive acute-on-chronic liver failure, and died of irreversible multiple organ dysfunction syndrome on 10 March 2020 (day 48 from the onset of the illness). Patient 2 and her family members requested that invasive rescue measures not be undertaken, and she died of acute respiratory distress syndrome on 22 March 2020 (day 40 from the onset of the illness). Patient 3 was discharged from the hospital on 11 March 2020 (day 29 from the onset of the illness) after the results of two real-time reverse-transcriptase polymerase-chain-reaction tests (nasopharyngeal swabs) taken $24 \mathrm{~h}$ apart were negative for SARS-CoV-2. 
Table 1 Clinical, laboratory, and radiographic findings at admission

\begin{tabular}{|c|c|c|c|}
\hline & Patient 1 & Patient 2 & Patient 3 \\
\hline \multicolumn{4}{|l|}{ Clinical characteristics } \\
\hline Age (years) & 53 & 75 & 87 \\
\hline Sex & Male & Female & Male \\
\hline Exposure history & $\begin{array}{r}\text { Travel to Wuhan } \\
\text { (Hubei, China) }\end{array}$ & $\begin{array}{r}\text { Travel to Wuhan } \\
\text { (Hubei, China) }\end{array}$ & $\begin{array}{l}\text { Contacted with } \\
\text { COVID-19 infected } \\
\text { patients }\end{array}$ \\
\hline \multicolumn{4}{|l|}{ Onset symptoms } \\
\hline Fever & Yes & Yes & Yes \\
\hline Diarrhea & No & No & Yes \\
\hline Anorexia & No & No & No \\
\hline Cough & Yes & No & Yes \\
\hline Sputum & Yes & No & Yes \\
\hline Shortness of breath & No & No & No \\
\hline Sore throat & No & No & No \\
\hline Dizziness & Yes & No & No \\
\hline Myalgia & Yes & No & No \\
\hline Headache & No & No & No \\
\hline Comorbidities & $\mathrm{T} 2 \mathrm{DM}$ & HTN & HTN, CKD \\
\hline Etiology of cirrhosis & Hepatitis B & Schistosomiasis & Alcoholic liver disease \\
\hline $\begin{array}{l}\text { Interval between onset and admis- } \\
\text { sion (days) }\end{array}$ & 3 & 30 & 9 \\
\hline Child-Pugh class & $\mathrm{C}$ & $\mathrm{C}$ & B \\
\hline MELD score & 15 & 14 & 23 \\
\hline \multicolumn{4}{|l|}{ Laboratory characteristics } \\
\hline White blood cell $\left(\times 10^{9} / \mathrm{L}\right)$ & 11.39 & 1 & 15.68 \\
\hline Neutrophils $\left(\times 10^{9} / \mathrm{L}\right)$ & 10.59 & 0.7 & 14.4 \\
\hline Lymphocyte $\left(\times 10^{9} / \mathrm{L}\right)$ & 0.36 & 0.17 & 0.74 \\
\hline Platelet $\left(\times 10^{9} / \mathrm{L}\right)$ & 77 & 28 & 74 \\
\hline ALT (U/L) & 26 & 44 & 19 \\
\hline AST (U/L) & 42 & 103 & 41 \\
\hline GGT (U/L) & 61 & 125 & 51 \\
\hline $\operatorname{ALP}(\mathrm{U} / \mathrm{L})$ & 62 & 148 & NA \\
\hline Total bilirubin $(\mu \mathrm{mol} / \mathrm{L})$ & 40.4 & 22.2 & 62.5 \\
\hline Direct bilirubin $(\mu \mathrm{mol} / \mathrm{L})$ & 12 & 15.3 & 38.1 \\
\hline Albumin $(\mathrm{g} / \mathrm{L})$ & 21.19 & 23.4 & 24.2 \\
\hline Lactate dehydrogenase (U/L) & 22 & 409 & 326 \\
\hline Blood urea nitrogen $(\mathrm{mmol} / \mathrm{L})$ & 11.5 & 9.3 & 14.35 \\
\hline Serum creatinine $(\mu \mathrm{mol} / \mathrm{L})$ & 66.2 & 98.2 & 192 \\
\hline APTT (s) & 28.2 & 55.5 & 48.3 \\
\hline Prothrombin time (s) & 17.2 & 17.7 & 17.4 \\
\hline International normalized ratio & 1.59 & 1.59 & 1.44 \\
\hline D-dimer $(\mu \mathrm{g} / \mathrm{L})$ & 0.66 & 18.05 & 2.1 \\
\hline C-reactive protein $(\mathrm{mg} / \mathrm{L})$ & 1.95 & 25.87 & 86.97 \\
\hline Procalcitonin (ng/mL) & 0.6 & 0.1 & 2.01 \\
\hline \multicolumn{4}{|l|}{ Chest CT evidence of pneumonia } \\
\hline Typical signs of viral infection & Yes & Yes & Yes \\
\hline
\end{tabular}

T2DM type 2 diabetes mellitus, HTN hypertension, CKD chronic kidney disease, MELD score Model for end-stage liver disease score, $A L T$ alanine aminotransferase, $A S T$ aspartate transaminase, $G G T \gamma$-glutamyl transpeptidase, $A L P$ alkaline Phosphatase, APTT activated partial thromboplastin time 
Table 2 Treatment, complications, and outcomes

\begin{tabular}{llll}
\hline & Patient 1 & Patient 2 & Patient 3 \\
\hline Treatment & & & \\
ICU admission & Yes & Yes & Yes \\
Antiviral treatment & Yes & Yes & Yes \\
Antibiotic treatment & Yes & Yes & Yes \\
Glucocorticoids & Yes & Yes & Yes \\
Intravenous immunoglobulin & Yes & Yes & Yes \\
Oxygen therapy & Yes & Yes & Yes \\
Noninvasive ventilation & No & Yes & Yes \\
Invasive mechanical ventilation & Yes & No & No \\
CRRT & Yes & No & No \\
ECMO & Yes & No & No \\
Complications during hospitalization & & \\
Bacterial pneumonia & Yes & No & Yes \\
Fungal pneumonia & Yes & No & No \\
Pleural effusion & Yes & Yes & Yes \\
Ascites & Yes & Yes & Yes \\
Melaena & Yes & No & Yes \\
Acute on chronic liver failure & Yes & No & No \\
Acute kidney injury & Yes & No & No \\
Shock & Yes & No & Yes \\
ARDS & Yes & Yes & No \\
Outcomes & Death & Death & Cure \\
Length of stay, days & 45 & 10 & 29 \\
\hline
\end{tabular}

$I C U$ intensive care unit, CRRT continuous renal replacement therapy, $E C M O$ extracorporeal membrane oxygenation, $A R D S$ acute respiratory distress syndrome

\section{Discussion}

Given that cirrhosis is one of the leading causes of death and illness globally [7], understanding how SARS-CoV-2 infection influences the clinical course in this setting is important. In this study, we report for the first time the clinical characteristics of three COVID-19 patients with pre-existing decompensated cirrhosis. Two patients with Child-Pugh C class disease died, while the patient with Child-Pugh Class B did not. Furthermore, the patient with the highest MELD score survived, while the ones with lower MELD scores did not. This raises the possibility that clinical decompensating events may be more important in predicting outcome of patients with COVID-19 and pre-existing cirrhosis.

COVID-19 patients with pre-existing decompensated cirrhosis seem to have a higher complication and mortality rate than those with COVID-19 alone [1-3]. In this study, all patients were admitted to the ICU and two of them died during hospitalization. A possible explanation for this might be the poorer immune function in patents with decompensated cirrhosis, since immunodeficiency is more prominent as the disease progresses [8]. Our data were consistent with previous work suggesting that patients with acute respiratory distress syndrome and cirrhosis, regardless of the disease stage, had worse outcomes than patients with acute respiratory distress syndrome without cirrhosis [9].

We were surprised by the outcome of patient 3 , who was considerably older than the other patients reported. However, he may have had better liver function than patients 1 and 2, given his lower Child-Pugh class and international normalized ratio. On the other hand, he had chronic kidney disease, which could be another risk factor for an adverse outcome of COVID-19. We recommend that for COVID-19 patients with pre-existing decompensated cirrhosis, while following the management principles of COVID-19, adequate nutritional support and proactive treatment to prevent decompensating events of liver cirrhosis are important.

We recognize that this study is limited by the small sample size. However, the death of 2 patients is notable. We expect that further studies with larger samples (COVID-Cirrhosis-CHESS, NCT04329559) will shed light on the clinical course of COVID-19 in patients with pre-existing cirrhosis.

In conclusion, we report here on the clinical course of COVID-19 patients with pre-existing decompensated cirrhosis. The data suggest that in patients with COVID-19, decompensated cirrhosis might be considered as a risk factor for a poor outcome.

Acknowledgements We thank the critical revision of Juan Gonzalez Abraldes (University of Alberta, Edmonton, Canada), Xavier Dray (Saint Antoine Hospital, APHP \& Sorbonne University, Paris, France), Andres Cardenas (Institute of Digestive Diseases and Metabolism, Hospital Clinic, University of Barcelona, Barcelona, Spain), Rino A. Gania (Dr. Cipto Mangunkusumo Hospital, Universitas Indonesia, Jakarta, Indonesia), Ashok Kumar Choudhury (Institute of Liver \& Biliary Sciences, New Delhi, India), and Necati Örmeci (Ankara University School of Medicine, Ankara, Turkey) and great support of members of the COVID-Cirrhosis-CHESS Group: Mingkai Chen (Renmin Hospital of Wuhan University, Wuhan), Zhengyan Wang (Suizhou Hospital, Hubei University of Medicine, Suizhou), Bin Xiong (Union Hospital, Tongji Medical College, Huazhong University of Science and Technology, Wuhan), Jianwen Wang (Wuhan Jinyintan Hospital, Wuhan), Yan Luo (Tianjin Haihe Hospital, Tianjin), Qing He (The Third People's Hospital of Shenzhen, Shenzhen), Guo Zhang (The People's Hospital of Guangxi Zhuang Autonomous Region, Nanning), Chuxiao Shao (Lishui Central Hospital, Lishui), Zhenhuai Chen (The People's Hospital of Baoding, Baoding), Dengxiang Liu (Xingtai People's Hospital, Xingtai), Shengqiang Zou (The Affiliated Third Hospital of Jiangsu University, Zhenjiang), Baoyi Ma (The People's Hospital of LinXia Hui Prefecture, Linxia), Ye Gu (The Sixth People's Hospital of Shenyang, Shenyang).

Author contributions Concept and design: XQ; acquisition of data: XL, ZW, HY, XL, JS, HX, and TL; interpretation of data: ZJ and FW; drafting of the manuscript: JW, YL, and XQ. Critical revision of the manuscript: SKS, DCR, TT, NK, and HM.

Funding Gansu Provincial COVID-19 Science and Technology Major Project. 


\section{Compliance with ethical standards}

Conflict of interest The authors have declared no conflict of interest related to the study.

\section{References}

1. Chen N, Zhou M, Dong X, Qu J, Gong F, Han Y, et al. Epidemiological and clinical characteristics of 99 cases of 2019 novel coronavirus pneumonia in Wuhan, China: a descriptive study. Lancet 2020;395(10223):507-513

2. Huang C, Wang Y, Li X, Ren L, Zhao J, Hu Y, et al. Clinical features of patients infected with 2019 novel coronavirus in Wuhan, China. Lancet 2020;395(10223):497-506

3. Guan WJ, Ni ZY, Hu Y, Liang WH, Ou CQ, He JX, et al. Clinical characteristics of coronavirus disease 2019 in China. N Engl J Med https://doi.org/10.1056/NEJMoa2002032

4. Zhang C, Shi L, Wang F. Liver injury in COVID-19: management and challenges. Lancet Gastroenterol Hepatol 2020;20:30057
5. European Association for the Study of the Liver. EASL Clinical Practice Guidelines for the management of patients with decompensated cirrhosis. J Hepatol 2018;69:406-460

6. Fukui H, Saito H, Ueno Y, Uto H, Obara K, Sakaida I, et al. Evidence-based clinical practice guidelines for liver cirrhosis 2015. J Gastroenterol 2016;51(7):629-50

7. GBD 2017 Cirrhosis Collaborators. The global, regional, and national burden of cirrhosis by cause in 195 countries and territories, 1990-2017: a systematic analysis for the Global Burden of Disease Study 2017. Lancet Gastroenterol Hepatol 2020;5(3):245-266

8. Albillos A, Lario M, Álvarez-Mon M. Cirrhosis-associated immune dysfunction: distinctive features and clinical relevance. J Hepatol 2014;61:1385-1396

9. Gacouin A, Locufier M, Uhel F, Letheulle J, Bouju P, Fillatre $\mathrm{P}$, et al. Liver cirrhosis is independently associated with 90-day mortality in ARDS patients. Shock 2016;2016(45):16-211

Publisher's Note Springer Nature remains neutral with regard to jurisdictional claims in published maps and institutional affiliations.

\section{Affiliations}

\section{Xiaolong $\mathrm{Qi}^{1} \oplus$ - Jitao Wang ${ }^{1} \cdot$ Xinyu $\mathrm{Li}^{2} \cdot$ Zhengyan Wang $^{3} \cdot$ Yanna Liu ${ }^{1} \cdot$ Hua Yang ${ }^{4}$ Xiaodan $\mathrm{Li}^{5}$. Jindong Shi ${ }^{6}$. Huihua Xiang ${ }^{7} \cdot$ Tao Liu $^{5}$. Norifumi Kawada ${ }^{8} \cdot$ Hitoshi Maruyama $^{9} \cdot$ Zicheng Jiang $^{10}$. Fengmei Wang ${ }^{11}$. Tetsuo Takehara ${ }^{12}$. Don C. Rockey ${ }^{13}$. Shiv Kumar Sarin ${ }^{14}$. for the COVID-Cirrhosis-CHESS Group}

1 CHESS (Chinese Portal Hypertension Diagnosis and Monitoring Study Group) Center, Institute of Portal Hypertension, The First Hospital of Lanzhou University, Lanzhou 730000, Gansu, China

2 Department of Infectious Disease, Shanghai Fifth People's Hospital of Fudan University, Shanghai, China

3 Departments of Respiratory Medicine, Suizhou Hospital, Hubei University of Medicine, Suizhou, Hubei, China

4 Departments of Respiratory Medicine, Minda Hospital Affiliated to Hubei University for Nationalities, Enshi, Hubei, China

5 Department of Infectious Disease, The Central Hospital of Enshi Tujia And Miao Autonomous Prefecture, Enshi, Hubei, China

6 Department of Respiratory Medicine, Shanghai Fifth People's Hospital of Fudan University, Shanghai, China

7 Department of Radiology, Minda Hospital Affiliated to Hubei University for Nationalities, Enshi, Hubei, China
8 Department of Hepatology, Graduate School of Medicine, Osaka City University, Osaka, Japan

9 Department of Gastroenterology, Juntendo University, Tokyo, Japan

10 Department of Infectious Disease, Ankang Central Hospital, Ankang, China

11 Department of Gastroenterology and Hepatology, Tianjin Second People's Hospital, Tianjin, China

12 Department of Gastroenterology and Hepatology, Osaka University Graduate School of Medicine, Osaka, Japan

13 Division of Gastroenterology and Hepatology, Medical University South Carolina, Charleston, SC, USA

14 Department of Hepatology, Institute of Liver and Biliary Sciences, New Delhi, India 